\title{
ADSORPTION OF PHOSPHONATES ON GYPSUM CRYSTALS
}

\author{
M.P.C. WEIJNEN * and G.M. VAN ROSMALEN \\ Department of Chemistry, Delft University of Technology, De Vries van Heystplantsoen 2, 2628 RZ Delft, The Netherlands
}

\begin{abstract}
The adsorption of phosphonate inhibitors at the crystal surface of gypsum has been determined from the decrease in phosphonate concentration of the bulk solution. The phosphonate was therefore oxidized to orthophosphate and spectrophotometrically determined as a molybdovanadophosphoric acid complex. The adsorption isotherms of AMDP at $\mathrm{pH} 5$ and HEDP at $\mathrm{pH}$ values of 5 and 7 were measured at $25^{\circ} \mathrm{C}$. From a comparison with the inhibitor effectiveness of both compounds during suspension growth experiments under similar conditions the surface coverage needed for growth inhibition was concluded to be $4-5 \%$. The growth process appears to be blocked when all active growth sites provided by the steps upon the crystal surface, are occupied by adsorbed phosphonate ions. The phosphonate adsorption process appears to be irreversible and almost instantaneously completed. Differences in inhibitor performance of AMDP and HEDP at $\mathrm{pH} \mathrm{5,} \mathrm{or} \mathrm{of} \mathrm{HEDP} \mathrm{at} \mathrm{pH} 5$ and 7, can be explained from a different surface coverage of the gypsum crystals.
\end{abstract}

\section{Introduction}

Among other mineral salts gypsum, $\mathrm{CaSO}_{4}$. $2 \mathrm{H}_{2} \mathrm{O}$, is known to be a notorious scalant in many industrial processes, such as desalination of brackish and seawater by reverse osmosis, oil and gas recovery, hydrometallurgical treatment of zinc ores and dairy production processes. Particularly in systems where the process flow proceeds through porous media like rock formations or membranes, scale deposition can only be prevented by means of chemical methods. A most successful scale prevention method uses the addition of scale inhibitors [1]. Phosphonate as well as polycarboxylate inhibitors are widely applied in industrial practice. Dosage of these compounds in the ppm range usually suffices to suppress unwanted mineral precipitation. Besides their remarkable effect on the crystallization kinetics, inhibitors also influence the habit and morphology of the crystals as well as their dispersive/agglomerative properties. Phosphonate inhibitors can thus be applied to improve the gypsum filterability in flue gas de-

* Present address: Koninklijke/Shell Laboratorium Amsterdam, P.O. Box 3003, Badhuisweg 3, 1003 AA Amsterdam, The Netherlands. sulfurization scrubbers, as has also been reported for citrates [2].

\section{Mechanism of adsorption}

Blockage of the mineral nucleation and growth process by trace amounts of an inhibitor in solution can only be ascribed to preferential adsorption onto developing nuclei and onto the crystal surface. Intriguing aspects of this adsorption process are the location of the inhibitor molecules or ions upon the crystal surface and the type of bonding involved. For barite as well as gypsum it has been reported that only a few percent of the crystal surface need to be covered with phosphonate inhibitor to achieve total blockage of the crystal growth process [3-5]. It is therefore generally assumed that relatively small inhibitor molecules, like most phosphonates, are preferentially adsorbed at the active growth sites upon the crystal surface. Active growth sites are provided by steps, originating either from dislocations or from twodimensional surface nucleation, and by kink sites in the steps. For the larger polycarboxylate inhibitors preferential adsorption along the steps is considered rather unlikely, because of the entropy loss involved in such an arrangement. However, even if

0022-0248/86/\$03.50 @ Elsevier Science Publishers B.V.

(North-Holland Physics Publishing Division) 
the polycarboxylates were randomly adsorbed upon the surface, the final result would be the same: the step propagation will be blocked as soon as the advancing steps are over their full length bounded by adsorbed inhibitor molecules.

The chemical bonding with the crystal surface is mainly accomplished through the anionic functional groups of the inhibitor. As reported previously [5], the remarkable increase in gypsum growth retardation at a given phosphonate inhibitor concentration with increasing $\mathrm{pH}$ values can quite accurately be predicted from the increasing concentration of inhibitor ions with at least one fully dissociated phosphonic acid group, $\mathrm{PO}_{3}^{2-}$, if the calculated $\mathrm{PO}_{3}^{2-}$ concentration is only based on the acid dissociation equilibrium, while the calcium complexation in the solution is neglected. The validity of this empirical rule indicates, that the complex formation equilibrium in the bulk of the solution is not representative for the equilibrium near the surface of the crystalline phase. The predictability of the phosphonate inhibitor effectiveness from its degree of dissociation points at a predominant role of the $\mathrm{PO}_{3}^{2-}$ groups, which can be considered responsible for the primary ionic interaction between the inhibitor ions and the crystal surface. Even in a comparison of phosphonate inhibitors with different molecular structures, the $\mathrm{PO}_{3}^{2-}$ concentration can still be used as a rough measure to predict the sequence of their growth retarding effect on gypsum [6].

A strong electrostatic interaction, as provided by the $\mathrm{PO}_{3}^{2-}$ groups, is primarily needed to induce the inhibitor ions to settle at the crystal surface, whereafter actual bond formation can occur. Once contact with the crystal surface has been established, the protonated acid groups, $\mathrm{PO}_{3} \mathrm{H}^{-}$, will be able to form stronger complexes with the calcium ions than the fully deprotonated acid groups. At very high $\mathrm{pH}$ values, where even the weakly acidic groups start to deprotonate, the phosphonate inhibitor effectiveness consequently starts to decrease $[7,8]$. The high sensitivity of gypsum for inhibitor ions with doubly charged anionic groups can be explained from the occurrence of a water sheet around the calcium ions in the gypsum surface. Owing to the high hydration energy of the calcium ions they will largely be shielded by water molecules, either originating from the crystal lattice or from the surrounding solution. Only inhibitor ions with highly charged anionic groups will probably be able to disrupt the strongly polarized water layer, thereby exposing the calcium ions underneath to strong coordinative bonding possibilities.

The fact that small carboxylate compounds are not effective as inhibitors can be due to their singly charged anionic groups. The high anionic charge density required to penetrate the water layer around the calcium ions, can only be attained by the presence of several dissociated carboxylic acid groups in one inhibitor molecule. At the same time a sufficient number of protonated carboxylic acid groups must remain to ensure strong coordinative bonding with the calcium ions in the surface $[9,10]$. To fulfill both demands of a high anionic charge density and a weak acid functionality over a wide range of $\mathrm{pH}$ values, polycarboxylate inhibitors need to contain a minimum of about 15 carboxylic acid groups per molecule, whereas most commercial phosphonate inhibitors contain only 3 to 5 phosphonic acid groups per molecule. Phosphonate inhibitors with less than 3 phosphonic acid groups per molecule invariably contain additional functional groups like carboxylic acid, hydroxyl or amino groups, which are also able to complex with the calcium ions in the gypsum crystal surface and/or to form hydrogen bridges with the sulfate ions or the crystal water molecules.

\section{Aim of the study}

The present investigation was performed to find out if the difference in inhibitor effectiveness between two phosphonate compounds, at the same inhibitor concentration and $\mathrm{pH}$ value, as well as the difference in inhibitor effectiveness of one given phosphonate concentration at different $\mathrm{pH}$ values, can be correlated with a different surface coverage of the gypsum crystals. Two bisphosphonate compounds were selected for this study: 1-hydroxy-ethane-1,1-bisphosphonic acid (HEDP) and amino-methane-bisphosphonic acid (AMDP). Both their adsorption at the gypsum crystal surface 
and their effect on the gypsum growth kinetics were determined, under equal conditions of temperature, $\mathrm{pH}$ value, inhibitor concentration and ionic strength, and compared to one another. The study was focussed on the influence of phosphonate inhibitors in pure gypsum solutions, but a few additional experiments were done in the presence of sodium chloride.

\section{Experimental}

Analytical grade chemicals and doubly distilled water were used. Precautions were taken to keep the solutions free from $\mathrm{CO}_{2}$. Supersaturated gypsum solutions were prepared from the more soluble calcium sulfate hemihydrate. Saturated gypsum solutions were obtained from slightly supersaturated solutions by adding a small amount of suspended, well-aged gypsum crystals. All solutions were passed through $0.22 \mu \mathrm{m}$ filters (Millipore). The phosphonate compounds were kindly donated by Henkel $\mathrm{GmbH}$. After addition of the phosphonate, if applied, the $\mathrm{pH}$ of the gypsum solutions was adjusted with minor amounts of $\mathrm{NaOH}$ or $\mathrm{H}_{2} \mathrm{SO}_{4}$ solutions. The calcium content of the solutions was checked by titration with EDTA.

The adsorption experiments were performed in saturated gypsum solutions at $25.00 \pm 0.05^{\circ} \mathrm{C}$. Each experiment was done in triplicate. The adsorption isotherms of AMDP and HEDP were both determined at a $\mathrm{pH}$ value of $5.00 \pm 0.02$ and the HEDP adsorption isotherm was also determined at $\mathrm{pH} 7.0 \pm 0.1$. In most of the adsorption experiments dry gypsum crystals (Merck) were used without any previous treatment. In some series of experiments these crystals were subjected to Ostwald-ripening before addition of the phosphonate. The specific surface area of the untreated and ripened crystals was determined from BET gas adsorption measurements to be $0.43 \pm 0.04$ and $0.36 \pm 0.03 \mathrm{~m}^{2} \mathrm{~g}^{-1}$ respectively (Quantasorb; gas mixtures $\mathrm{N}_{2} / \mathrm{He} 5 / 95,10 / 90,15 / 85$ and $20 / 80$ ).

At the start of the adsorption experiments an accurately weighed amount of dry gypsum crystals was added to $50.00 \mathrm{ml}$ of saturated solution, after adjustment of the phosphonate concentration and $\mathrm{pH}$ value. The solid/liquid ratio of the suspensions was about $50 \mathrm{mg} \mathrm{ml}^{-1}$. The suspensions were gently shaken for $48 \mathrm{~h}$ in a thermostated bath, thus providing ample time to reach the adsorption equilibrium state [4,5]. After settling of the crystals a $25.00 \mathrm{ml}$ sample was taken from the supernatant solution in order to determine the residual phosphonate concentration. The phosphonate was therefore oxidized using the persulfate-UV method [11]. After addition of the required amount of $\mathrm{K}_{2} \mathrm{~S}_{2} \mathrm{O}_{8}$ (Baker) the solution was exposed to UV radiation ( $254 \mathrm{~nm}$ ) for $20 \mathrm{~min}$ at $50^{\circ} \mathrm{C}$. This temperature was chosen to ensure a high intensity of the immersed Hg UV lamp (Pen Ray lamp, Ultra-Violet Products), needed to obtain rapid and complete conversion to orthophosphate. After oxidation and cooling a $10.00 \mathrm{ml}$ sample from the solution was diluted with $0.42 \mathrm{ml}$ of a $2.5 \mathrm{M} \mathrm{HCl}$ solution and $10.00 \mathrm{ml}$ of a freshly diluted mixed reagent solution containing $\mathrm{NH}_{4} \mathrm{VO}_{3}$ (Baker), $\left(\mathrm{NH}_{4}\right)_{6} \mathrm{Mo}_{7} \mathrm{O}_{24}$ (Fluka) and $\mathrm{HCl}(\mathrm{BDH}$ Chemicals), prepared as described by Michelsen [12]. After 2 min the resulting molybdo/vanado/ phosphoric acid complex was determined spectrophotometrically by measuring its absorbance at $316 \mathrm{~nm}$, using a Perkin-Elmer $552 \mathrm{~S}$ double beam spectrophotometer and $1 \mathrm{~cm}$ quartz cells. The corresponding phosphonate concentration was derived from an absorbance versus concentration calibration curve. A reference calibration curve was made for $\mathrm{KH}_{2} \mathrm{PO}_{4}$ (Merck) solutions. As a check on the initial phosphonate concentration in each series of experiments saturated gypsum solutions with the same phosphonate concentration, but without crystals, were subjected to the same equilibrating and measuring procedure. No evidence was found for adsorption of AMDP or HEDP onto the glassware.

To study the possible influence of Ostwald ripening on the measured phosphonate adsorption levels, additional series of experiments were performed, where the Ostwald-ripening process and the phosphonate adsorption process were not allowed to occur simultaneously. In these experiments an accurately weighed amount of dry crystals was added to $50.00 \mathrm{ml}$ of water at the desired $\mathrm{pH}$ value. The mass of crystals was chosen 
such as to reach the same solid/liquid ratio of 50 $\mathrm{mg} \mathrm{ml}^{-1}$ after establishment of the gypsum equilibrium concentration by dissolution of the smallest crystals. After two days of gentle shaking in the thermostat bath at $25^{\circ} \mathrm{C}$ the Ostwald-ripening process could be assumed to be almost completed [13]. The crystals were allowed to settle and 25.00 $\mathrm{ml}$ of the supernatant solution was decanted and replaced by the same volume of a saturated gypsum solution with a known phosphonate concentration and the desired $\mathrm{pH}$ value. Thereafter the same equilibrating and measuring procedure was followed as described for the unripened crystals.

Suspension growth experiments were performed to study the gypsum growth kinetics. Well-defined seed crystals, prepared as described in ref. [14] and aged for at least three months, were used to initiate the growth process. Their specific surface area was determined to be $0.30 \pm$ $0.05 \mathrm{~m}^{2} \mathrm{~g}^{-1}$. During outgrowth of the gypsum seed crystals the composition of the bulk solution was kept constant by the controlled addition of a higher supersaturated solution with the same temperature, $\mathrm{pH}$ and additive concentration as the bulk solution. The experimental method and equipment have been detailed in refs. [5,15]. All growth experiments were performed at $25.00 \pm$ $0.01^{\circ} \mathrm{C}$. The solid/liquid ratio of the suspensions was about $1 \mathrm{mg} \mathrm{ml}^{-1}$. The inhibitor effectiveness of both HEDP and AMDP was determined at a phosphonate concentration of $10^{-6} \mathrm{M}$, a relative supersaturation of $20.0 \pm 0.1 \%$ and a $\mathrm{pH}$ value of $5.00 \pm 0.01$, while the HEDP effectiveness was also determined at $\mathrm{pH} 7.00 \pm 0.05$. Besides, the effectiveness of $10^{-6} \mathrm{M}$ and $10^{-5} \mathrm{M}$ HEDP was studied both in the absence and presence of 0.025 $\mathrm{M} \mathrm{NaCl}$, at a relative supersaturation of $15.0 \pm$ $0.2 \%$ and $\mathrm{pH} 5.00 \pm 0.01$.

For comparison with the growth experiments some adsorption experiments with HEDP at $\mathrm{pH} 5$ were also performed in the presence of $0.025 \mathrm{M}$ $\mathrm{NaCl}$. A few adsorption experiments were further performed in supersaturated gypsum solutions.

\section{Analysis of the experiments}

From the difference between the initial phosphonate concentration in the bulk solution and the residual phosphonate concentrate after completion of the adsorption process, the amount of phosphonate adsorbed onto the gypsum crystals can be calculated. Since an accurately weighed amount of crystals with a known specific surface area was used, the total surface area available for adsorption is also known. If the adsorption process was preceded by Ostwald ripening, the mass of added gypsum crystals is corrected for the amount dissolved in $50 \mathrm{ml}$ of water at $25^{\circ} \mathrm{C}(131$ $\mathrm{mg}$ ), and the proper value for the specific surface area is used. From the amount of phosphonate adsorbed per unit surface area of the crystals, the percentage of the gypsum crystal surface covered with inhibitor molecules can be calculated, assuming that one AMDP or HEDP molecule occupies an area of $50 \AA^{2}$. The $\mathrm{Na}_{2} \mathrm{H}_{2} \mathrm{HEDP} \cdot 4 \mathrm{H}_{2} \mathrm{O}$ crystal structure data reported by Barnett and Strickland [16] indicate that the surface area of the ${ }_{-} \mathrm{C}\left(\mathrm{PO}_{3} \mathrm{H}_{2}\right)_{2}$ part of the molecule is about $20 \AA^{2}$. Including a correction for the inhibiting effect on adjacent calcium ions in the gypsum crystal surface, the effective surface area of one adsorbed HEDP molecule is estimated to be $50 \AA^{2}$. Since the AMDP and HEDP molecules hardly differ in size and since both molecules are expected to adopt the same conformation upon the crystal surface, owing to their similar bonding possibilities in a similar configuration, the effective surface area per adsorbed AMDP molecule is also estimated to be $50 \AA^{2}$.

The analysis of the growth data is detailed in ref. [5]. Only a few essential definitions are given here: the outgrowth of the seed crystals is defined as their total volume at time $t$, divided by their original volume; the "normalized" overall growth rate of the crystals is defined as the time derivative of their total volume, divided by their original volume; the effectiveness of a given inhibitor concentration is defined as the ratio of the "normalized" overall growth rates in the absence and in the presence of the inhibitor, under equal growth conditions.

\section{Results and discussion}

Both the AMDP and HEDP calibration curves, determined in the concentration range of $10^{-6}-2$ 
$\times 10^{-5} \mathrm{M}$ AMDP and $10^{-6}-5 \times 10^{-5} \mathrm{M}$ HEDP, as well as the $\mathrm{KH}_{2} \mathrm{PO}_{4}$ calibration curve, determined in the range of $5 \times 10^{-6}-8 \times 10^{-5} \mathrm{M}$ $\mathrm{KH}_{2} \mathrm{PO}_{4}$, show a linear relationship between the measured absorbance of the molybdo/vanado/ phosphoric acid complex at $316 \mathrm{~nm}, A_{316}$, and the corresponding orthophosphate concentration, $\left[\mathrm{PO}_{4}^{3-}\right](\mathrm{mol} / \mathrm{l})$. This linear relationship and the correlation coefficient $r$ are given by:

$A_{316}=1.002 \times 10^{4}\left[\mathrm{PO}_{4}^{3-}\right]+4.0 \times 10^{-4}$,

with $r=0.9999$, for $\mathrm{KH}_{2} \mathrm{PO}_{4}$,

$A_{316}=0.970 \times 10^{4}\left[\mathrm{PO}_{4}^{3-}\right]+2.3 \times 10^{-2}$,

with $r=0.9995$, for HEDP,

$A_{316}=0.999 \times 10^{4}\left[\mathrm{PO}_{4}^{3-}\right]+5.1 \times 10^{-3}$,

with $r=0.9997$, for AMDP.

Beer's law is thus obeyed in the examined concentration range. For the accurate determination of higher phosphate concentrations, $>3.1 \mathrm{mg} \mathrm{P}$ $1^{-1}$, the orthophosphate solution obtained after persulfate-UV treatment was diluted $1: 2$, instead of $1: 1$, with the diluted mixed reagent solution. Both the AMDP and HEDP calibration curves practically coincide with the $\mathrm{KH}_{2} \mathrm{PO}_{4}$ calibration curve. This result proves that 20 minutes persulfate-UV treatment at $50^{\circ} \mathrm{C}$ is sufficient to achieve quantitative conversion of the phosphonates to orthophosphate.

The HEDP adsorption isotherm at $\mathrm{pH} 5$ is shown in fig. 1, where the percentage of the gypsum surface covered with HEDP is plotted versus the residual HEDP concentration in solution after establishment of the adsorption equilibrium. The given surface coverage data were obtained for untreated gypsum crystals as well as for crystals which were subjected to Ostwald ripening before addition of HEDP. The almost identical adsorption isotherms show two plateaus: the first one, at less than $1 \%$ surface coverage, is found between equilibrium concentrations of $10^{-6} \mathrm{M}$ and about $10^{-5} \mathrm{M}$ HEDP, and the second one at $5.0 \pm 0.5 \%$ surface coverage occurs between $5 \times 10^{-5} \mathrm{M}$ and $8 \times 10^{-5} \mathrm{M}$ HEDP. The effect of Ostwald-ripening is clearly illustrated from the surface coverage data measured at the first adsorption plateau. At low HEDP concentrations $<10^{-5} \mathrm{M}$ a small, but significant difference in adsorption on the untreated and ripened crystals is observed: the ripened crystals exhibit an adsorption plateau at $0.4 \pm 0.2 \%$ surface coverage, while a higher per-

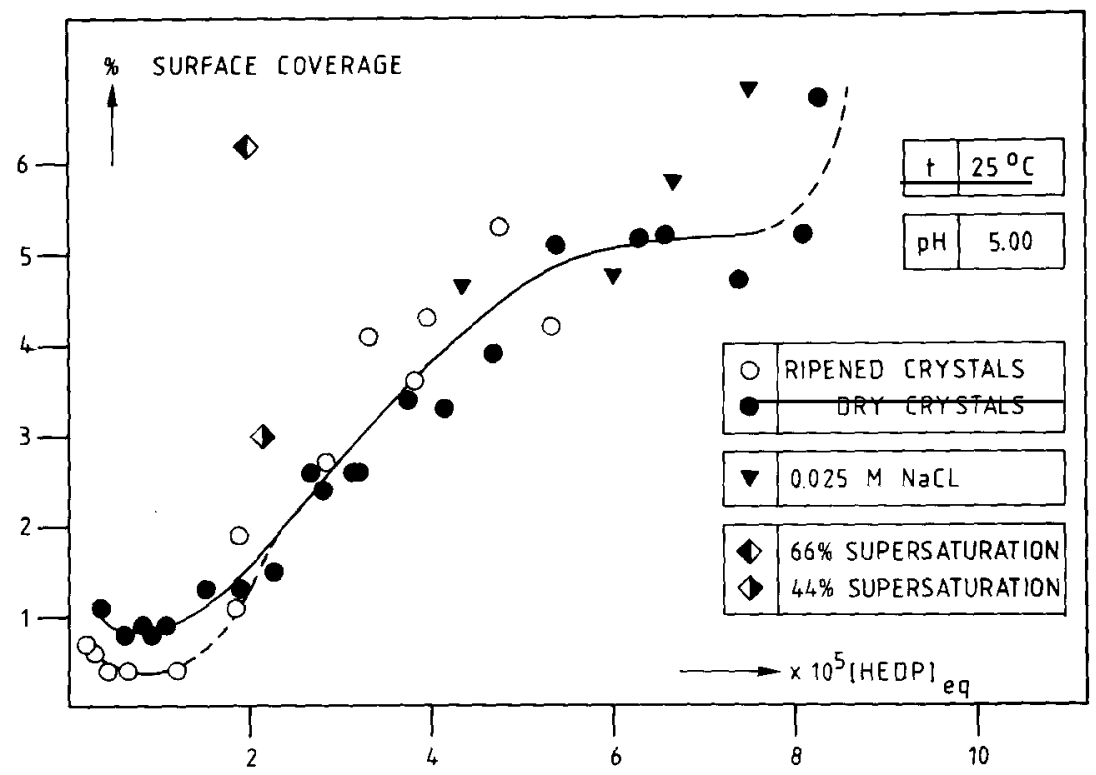

Fig. 1. Adsorption of HEDP on untreated and ripened gypsum crystals at pH 5; percentage of the gypsum surface covered with HEDP versus the HEDP equilibrium concentration in solution. 
centage, namely $0.8 \pm 0.2 \%$, of the untreated crystal surface seems to be covered with HEDP. The seemingly higher surface coverage of the untreated gypsum crystals can be explained from the occurrence of Ostwald-ripening simultaneously with the adsorption process. The solubility of gypsum noticeably increases with decreasing size of the crystals below $2 \mu \mathrm{m}$, as reported by Hulett [17], while Dundon and Mack [18] later corrected this "critical" diameter to about $0.5 \mu \mathrm{m}$. A small fraction of the added dry gypsum crystals will thus dissolve and recrystallize upon the larger crystals, thereby causing incorporation of already adsorbed HEDP and necessitating extra HEDP adsorption upon the newly formed crystal surface. The Ostwald-ripening process proceeds until only crystals with a diameter $>0.5 \mu \mathrm{m}$ remain in the suspension. Due to the relatively high solubility of gypsum this process is almost completed after 48 $h$ [13]. The surface coverage data given for the ripened crystals, where $48 \mathrm{~h}$ were allowed for Ostwald ripening before adsorption of HEDP could occur, can thus be assumed to represent the true adsorption equilibrium, whereas the surface coverage data given for the untreated crystals obviously are too high owing to incorporation of HEDP during the ripening process. At HEDP concentrations $>0.810^{-5} \mathrm{M}$, however, no significant difference in surface coverage of the untreated and ripened crystals can be found anymore. Apparently a surface coverage of $>1 \%$ is already sufficient to block the recrystallization process of the smallest crystals.

The occurrence of two plateaus in the HEDP adsorption isotherm at $\mathrm{pH} 5$ indicates a heterogeneity of the adsorption sites upon the gypsum crystal surface. Although according to thermodynamics the crystal surface should be perfectly smooth in its saturated solution, it is for kinetic reasons very unlikely that any mineral surface ever reaches the thermodynamic equilibrium state, unless extremely long ripening periods are applied. In contrast with Ostwald ripening, which could be referred to as "macro-ripening", the smoothing of the crystal surface is a "micro-ripening" process occurring on a considerably smaller scale. Gypsum is known to grow according to a spiral growth mechanism up to supersaturations of at least $70 \%$
$[14,19]$. Although the concentration history of the applied gypsum crystals is not known, it can nevertheless be expected that their surface exhibits a "frozen" spiral pattern. Three different types of surface sites can thus be distinguished: the flat parts of the crystal surface between the spiral steps, the steps and the kink sites in the steps. Both micro- and macro-steps can occur, the latter ones due to, e.g., step bunching at high supersaturations during the precipitation process of the gypsum crystals.

The plateaus in the HEDP adsorption isotherm cannot without further preface be assigned to preferential adsorption of HEDP onto specific types of surface sites, since it is neither known, if with increasing HEDP concentrations $>8.10^{-5}$ $M$ another adsorption plateau is encountered, nor which maximum surface coverage is finally attained. However, recently performed calculations of the step and kink density upon the various gypsum crystal faces revealed, that an overall surface coverage of $<1 \%$ ought to be sufficient to block all available kink sites, while a coverage of $2-4 \%$ suffices to block all steps on the entire surface of the gypsum crystals [20]. In these calculations the spiral pattern was assumed to be established at a supersaturation of $30 \%$ and the growth process was assumed to be dominated by single growth spirals. The surface area ratio of the various crystallographic faces, substituted in the calculation of the overall surface coverage, was derived from scanning electron micrographs of the gypsum seed crystals applied in the suspension growth experiments. Since the size distribution and the specific surface area of the gypsum crystals used in the adsorption experiments are not largely different from the seed crystals used in the growth experiments, the conclusion seems justified, that the plateau at $0.4 \%$ surface coverage corresponds with full occupation of the available kink sites and the second plateau at $5 \%$ coverage with full occupation of the total microstep length on the gypsum surface.

The adsorption isotherm of HEDP at $\mathrm{pH} \mathrm{5,} \mathrm{as}$ presented in fig. 1, qualitatively agrees with the adsorption isotherm determined by Gill and Nancollas [4] for the adsorption of triethylenediaminetetra(methylenephosphonic acid), 
TENTMP, on gypsum at pH 5.63 and $25^{\circ} \mathrm{C}$. In this case also two plateaus were observed, the first one at a surface coverage of less than $10^{-8} \mathrm{~mol}$ $\mathrm{g}^{-1}$ and the second one slightly above $3 \times 10^{-8}$ mol TENTMP per gram solid. According to the authors these plateaus correspond to surface coverages of $<4 \%$ and approximately $9 \%$ respectively. The substantially higher level of both plateaus in comparison with the plateau levels observed in fig. 1 is rather surprising, since the specific surface area of the gypsum crystals used by Gill and Nancollas, $0.4 \mathrm{~m}^{2} \mathrm{~g}^{-1}$, is about the same as for the crystals used in the present investigation, as can also be expected for the crystal size. Closer examination of their calculations, however, reveals that they substituted $200 \AA^{2}$ for the effective surface area of one TENTMP molecule, assuming $50 \AA^{2}$ to be the effective surface area of one phosphonate group. As can be concluded from the HEDP crystal structure data given in ref. [16], the surface area of one phosphonate group is close to $10 \AA^{2}$. Taking the inhibitory effect on adjacent calcium ions in the gypsum crystal surface into account, it seems reasonable to assume a value of $25 \AA^{2}$ for the effective surface area of one phosphonate group and a value of $100 \AA^{2}$ for the effective surface area of one TENTMP molecule. Substituting this value of $100 \AA^{2}$ in the calculation of the surface coverage percentage from the adsorption data reported for the plateaus in the TENTMP adsorption isotherm, leads to surface coverages of less than $1.5 \%$ and about $5 \%$ for the first and second plateaus respectively. This result is in good agreement with the present work. The first TENTMP adsorption plateau may be slightly enhanced due to Ostwald ripening, since Gill and Nancollas used dry crystals in their adsorption experiments.

Fig. 1 further presents the results of some additional adsorption experiments in solutions containing $0.025 \mathrm{M} \mathrm{NaCl}$. It follows that the adsorption of HEDP at pH 5 is not noticeably affected by this low $\mathrm{NaCl}$ concentration. On the contrary, Gill and Nancollas [4] report a substantial increase in TENTMP adsorption with increasing $\mathrm{NaCl}$ concentrations, until an almost constant value is reached at $0.2 \mathrm{M} \mathrm{NaCl}$. The same phenomenon of increased adsorption at high ionic strength has been reported for polyelectrolytes $[21,22]$. This increased adsorption, however, is not accompanied by an increased inhibitor effectiveness, as it is well-known from industrial practice that the inhibitor dosage levels needed to block mineral precipitation, are considerably enhanced in high ionic strength solutions. Apparently another kind of adsorption, so called low-affinity adsorption, prevails under such conditions. At high ionic strength the dissociated acid groups of the inhibitor will largely be shielded by the many other ions in solution. The electrostatic interaction between the inhibitor ions and the crystal surface is thus drastically weakened and the inhibitor ions will more or less behave like non-ionic adsorbants. Since those inhibitor ions are no longer able to disrupt the water layer around the calcium ions, strong coordinative bonds cannot be formed, and the loosely adherent inhibitor ions will easily be displaced by new crystal growth units impinging on the crystal surface.

Some adsorption experiments with HEDP at $\mathrm{pH} 5$ were performed in supersaturated gypsum solutions, at initial supersaturations of $44 \%$ and $66 \%$. The resulting increase in surface coverage, as can be observed in fig. 1, can partly be due to the increase in step density on the gypsum surface with increasing supersaturation. However, since the HEDP concentration prevailing during these adsorption experiments was not sufficient to block the gypsum growth process, the seeming increase in surface coverage is probably largely due to incorporation of adsorbed inhibitor ions during outgrowth of the added gypsum crystals to respectively $102.3 \%$ and $103.5 \%$ of their original weight.

In contrast with the HEDP adsorption isotherm at $\mathrm{pH} 5$, the plateaus in the TENTMP adsorption isotherm at pH5.63 [4] are reached at considerably lower equilibrium concentrations: the first plateau starts around $10^{-7} \mathrm{M}$ TENTMP and the second one around $10^{-6} \mathrm{M}$ TENTMP. Apparently TENTMP is more easily adsorbed than HEDP, resulting in a TENTMP adsorption isotherm shifted to lower equilibrium concentrations and compressed to a smaller concentration range, as compared with the HEDP adsorption isotherm. The same tendency is observed, when the HEDP adsorption isotherm at $\mathrm{pH} 7$ is compared with the 


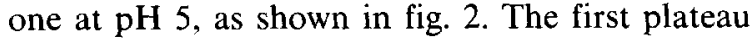
in the HEDP adsorption isotherm can hardly be detected anymore at $\mathrm{pH} 7$, since the applied method to determine the phosphonate concentration is not suited for accurate determination of HEDP concentrations $<10^{-6} \mathrm{~N}$. The second plateau, corresponding with a surface coverage of $4.0 \pm 0.5 \%$, is found at an equilibrium concentration of about $10^{-5} \mathrm{M}$ at $\mathrm{pH} 7$, whereas at $\mathrm{pH} 5$ a concentration of about $5.10^{-5} \mathrm{M}$ HEDP is needed to reach the second plateau.

From fig. 2 it is clear that the surface coverage of the gypsum crystals at a given HEDP concentration is substantially higher at a $\mathrm{pH}$ value of

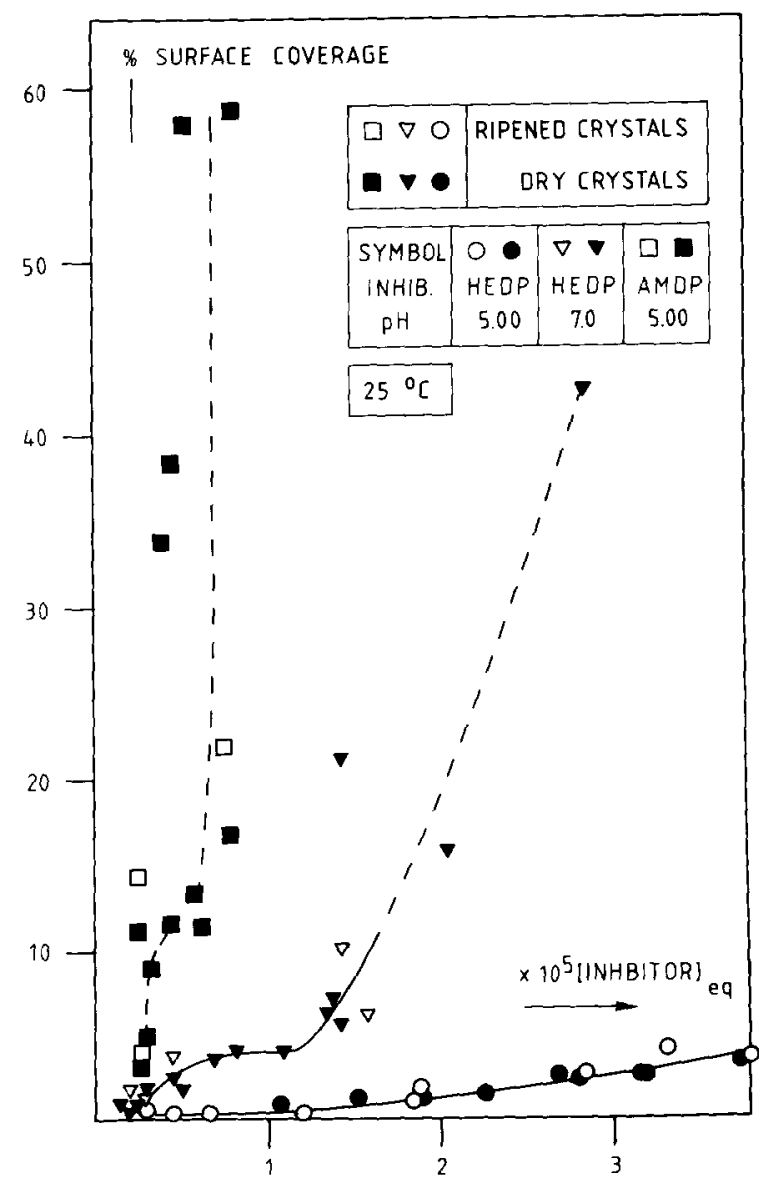

Fig. 2. Adsorption of AMDP at $\mathrm{pH} 5$ and HEDP at $\mathrm{pH} 5$ and 7 on gypsum; percentage of the gypsum surface covered with inhibitor versus the solution equilibrium concentration of the inhibitor.
7 than at $\mathrm{pH}$ 5. It is therefore surprising that, according to Gill and Nancollas [4], the adsorption of TENTMP on gypsum is hardly affected by the $\mathrm{pH}$ of the solution. This can perhaps be explained from their use of a relatively high TENTMP concentration of about $2 \times 10^{-6} \mathrm{M}$ in their study of the $\mathrm{pH}$ influence on adsorption. At this concentration, corresponding with the second plateau in the TENTMP adsorption isotherm at pH 5.63 and probably with full coverage of the active growth sites, a further increase in $\mathrm{pH}$ apparently does not result in additional TENTMP adsorption on sites energetically less favourable than the active growth sites. Even at $\mathrm{pH} 1$ the reported surface coverage with TENTMP is only slightly lower than the maximum value found between $\mathrm{pH} 4$ and 7. Although this result seems to contradict the general experience, that phosphonate inhibitors at accepted dosage levels are not effective in gypsum growth retardation at $\mathrm{pH}$ values $<3$, it can be explained from the occurrence of low-affinity adsorption. Since the phosphonates are practically undissociated at these low pH values, they behave like non-ionic adsorbants, as also described for high ionic strength solutions. With increasing $\mathrm{pH}$ values $>10$ Gill and Nancollas observed a tendency towards decreasing TENTMP adsorption levels, in accordance with the practical experience of a decreasing phosphonate inhibitor effectiveness at very high $\mathrm{pH}$ values, which can be owed to a lack of protonated acid groups and thus to a lack of strong coordinative bonding possibilities.

Fig. 2 also shows that the AMDP adsorption isotherm at $\mathrm{pH} 5$ is situated at a higher surface coverage level than both HEDP adsorption isotherms at $\mathrm{pH} 5$ and 7. The AMDP adsorption isotherm exhibits no sign of adsorption plateaus comparable to those in the HEDP isotherms. The inflexion point occurring at about $12 \%$ surface coverage may not be significant. Comparison of the surface coverages measured on untreated and ripened gypsum crystals with AMDP at $\mathrm{pH} 5$ and with HEDP at $\mathrm{pH} 7$, also plotted in fig. 2, does not reveal significant differences between both types of crystals. Even at the lowest phosphonate concentrations used, the established surface coverage appears sufficient to block the Ostwald-ripen- 
ing process, as could be expected from the results discussed for HEDP at $\mathrm{pH}$ 5. The similar adsorption behaviour of the untreated and ripened crystals at surface coverages $>1 \%$ further indicates, that the phosphonate adsorption rate is very high, as compared to the rate at which Ostwald ripening occurs. Additional adsorption experi-

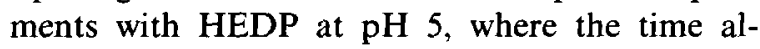
lowed for equilibration was reduced to $5 \mathrm{~h}$ or $1 \mathrm{~h}$ only, indeed showed the same results as obtained after $48 \mathrm{~h}$ equilibration time. It should further be noted, that the adsorption of phosphonate onto the gypsum crystal surface seems to be an irreversible process. When gypsum crystals covered with HEDP at $\mathrm{pH} 5$ or 7 , were resuspended in a pure saturated gypsum solution, no release of adsorbed phosphonate to the surrounding solution could be detected, even after several days.

\section{Comparison of adsorption and growth experi- ments}

The results of growth experiments performed in the presence of $10^{-6} \mathrm{M}$ and $10^{-5} \mathrm{M}$ HEDP, both in the presence and absence of $0.025 \mathrm{M} \mathrm{NaCl}$, and the corresponding blanks are shown in fig. 3, where the inhibitor effectiveness is plotted versus the outgrowth of the seed crystals. From this figure it can be concluded, that the effectiveness of a given HEDP concentration in retarding the gypsum growth kinetics is lower in the presence than in the absence of $\mathrm{NaCl}$, at the same relative supersaturation of $15 \%$. As shown in fig. 1, however, the surface coverage measured in the presence of $0.025 \mathrm{M} \mathrm{NaCl}$ is about the same as in pure gypsum solutions, indicating that this $\mathrm{NaCl}$ concentration is too low to cause enhanced adsorption of the low-affinity type. The decrease in HEDP inhibitor effectiveness must therefore be due to the substantially larger supply of gypsum growth units from the $\mathrm{NaCl}$ containing bulk solution. The gypsum equilibrium concentration at $25^{\circ} \mathrm{C}$ in pure water was determined to be 0.01522 mol $1^{-1}$, while the equilibrium concentration in $0.025 \mathrm{M} \mathrm{NaCl}$ is $0.01743 \mathrm{~mol} \mathrm{l}^{-1}$, as determined from titration as well as by interpolation from the solubility data given by Marshall and Slusher [23].

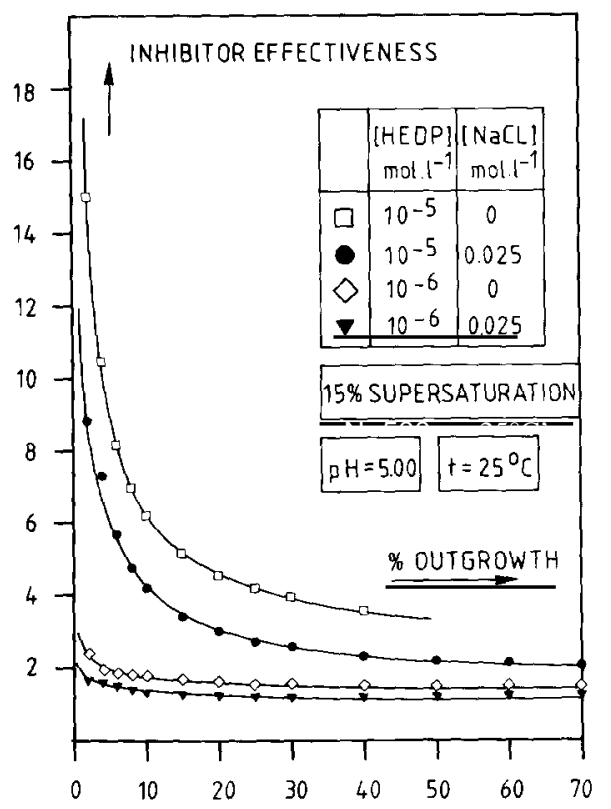

Fig. 3. Growth retardation of gypsum by HEDP in the absence and in the presence of $0.025 \mathrm{M} \mathrm{NaCl}$, for two HEDP concentrations at $\mathrm{pH} 5$; inhibitor effectiveness versus the outgrowth of the seed crystals.

The influence of $\mathrm{pH}$ on the inhibitor effectiveness of a given HEDP concentration has been elucidated in ref. [5]. For $\mathrm{pH}$ values of 5 and 7, the inhibitor effectiveness of $10^{-6} \mathrm{M}$ HEDP at a constant supersaturation of $20 \%$ is plotted in fig. 4 versus the outgrowth of the seed crystals. The substantially higher surface coverage of the gypsum crystals with HEDP at $\mathrm{pH} 7$ in comparison with $\mathrm{pH} 5$, as already shown in fig. 2 , turns out to correspond with a substantially higher effectiveness of HEDP in retarding the gypsum growth kinetics at $\mathrm{pH} 7$. The same result is found when the inhibitor effectiveness of $10^{-6} \mathrm{M}$ AMDP at $\mathrm{pH} 5$ is compared with the effectiveness of HEDP at $\mathrm{pH} 5$ and 7 , at the same inhibitor concentration and supersaturation. Comparison of figs. 2 and 4 shows, that the higher effectiveness of AMDP can again be correlated with a higher surface coverage of the gypsum crystals.

The inhibitor concentrations needed to block the gypsum growth process at supersaturations $<30 \%$, are about $6 \times 10^{-5} \mathrm{M}$ HEDP at $\mathrm{pH} 5$, $10^{-5}$ M HEDP at pH 7 and about $(2-3) \times 10^{-6}$ 


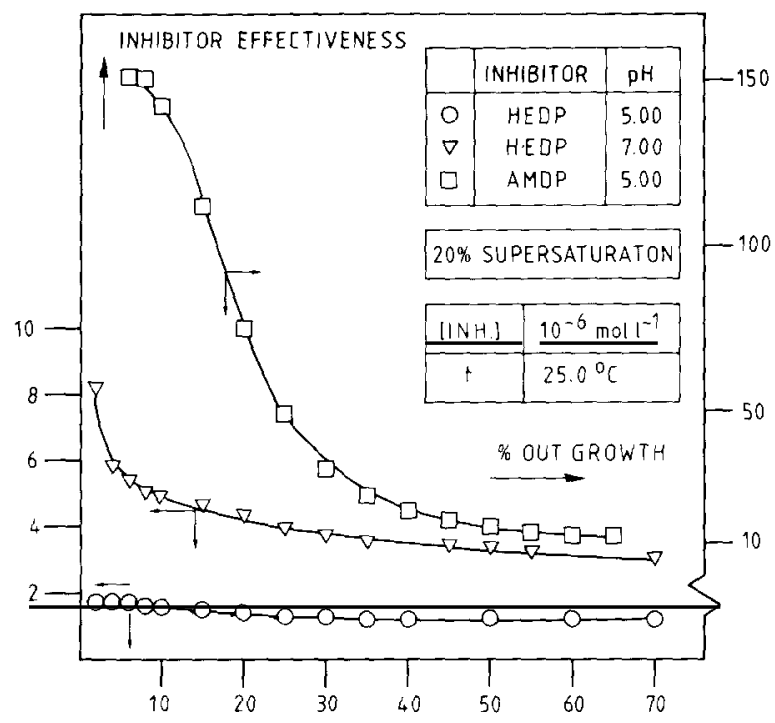

Fig. 4. Growth retardation of gypsum by AMDP at $\mathrm{pH} 5$ and HEDP at $\mathrm{pH} 5$ and 7 , at the same inhibitor concentration of $10^{-6} \mathrm{M}$; inhibitor effectiveness versus the outgrowth of the seed crystals.

M AMDP at pH 5. In all three cases the adsorption measurements reveal a corresponding surface coverage between 4 and 5\%. Since the second

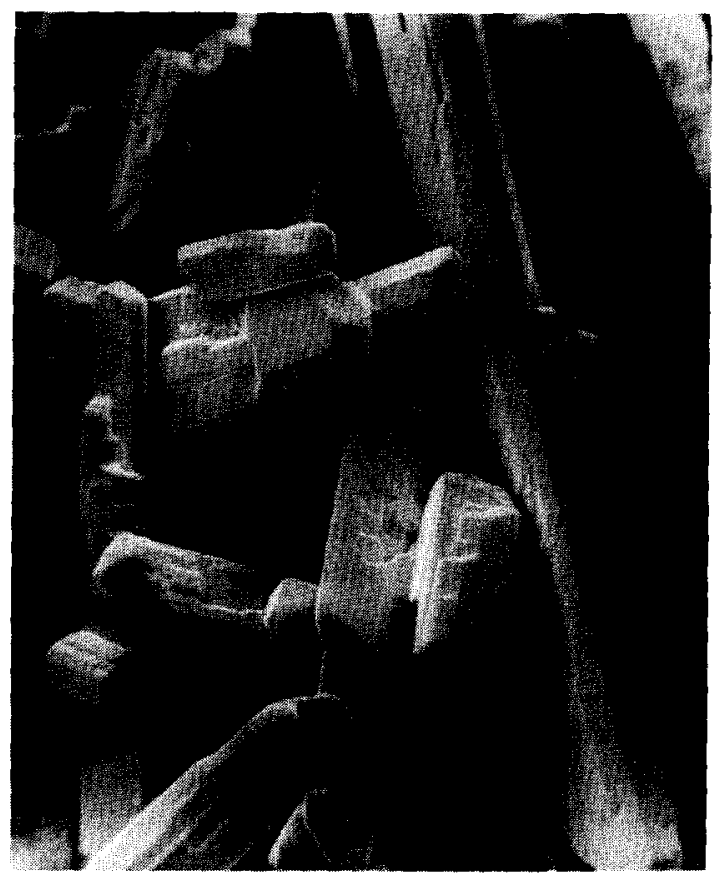

Fig. 5. SEM view of the gypsum seed crystals applied in the suspension growth experiments (magnification $725 \times$ ).

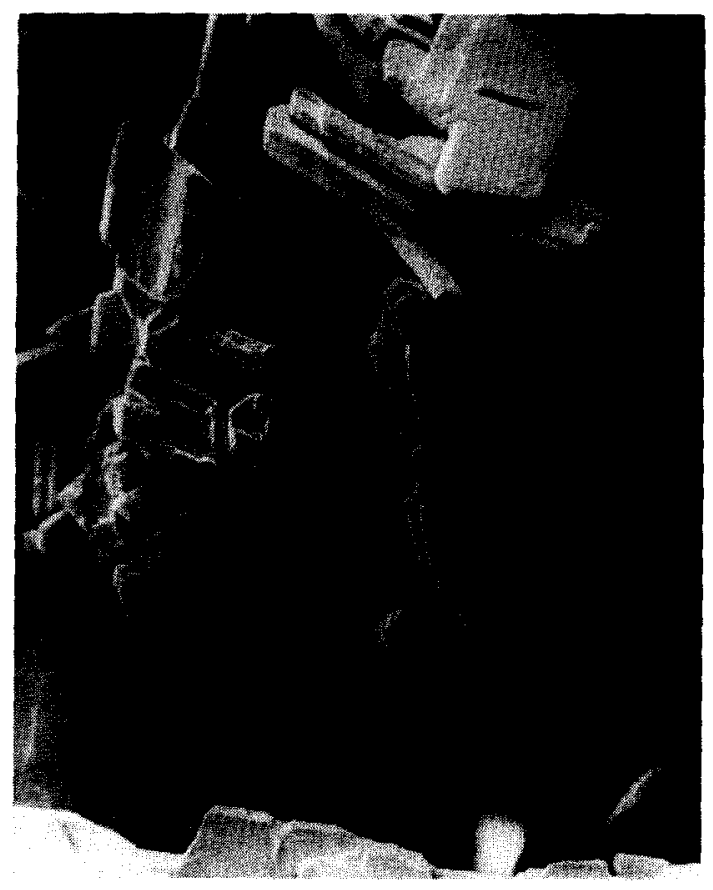

Fig. 6. SEM view of the gypsum crystals (Merck) applied in the adsorption experiments (magnification $725 \times$ ).

plateau in the HEDP adsorption isotherms at $\mathrm{pH}$ 5 and 7 is found at the same surface coverage, and since this value agrees with the theoretical surface coverage, calculated for occupation of the total microstep length on the gypsum crystal surface at a supersaturation of $30 \%$, strong evidence is obtained for the hypothesis that phosphonate inhibitors are preferentially adsorbed at the active growth sites upon the gypsum surface. As mentioned before, large differences in adsorption behaviour between the gypsum seed crystals used in the growth experiments and the crystals used in the adsorption experiments are not to be expected. Preliminary adsorption experiments on dried gypsum seed crystals have so far confirmed this expectation. Even after three months of aging the seed crystal surface still shows many irregularities, rendering them more or less comparable to the rather rough gypsum crystals (Merck) used in the adsorption experiments. This is illustrated by scanning electron micrographs of both crystal batches, given in figs. 5 and 6.

In fig. 7 the growth kinetics of "normal" seed 
crystals and seed crystals pretreated with HEDP are compared, during outgrowth in the presence as well as in the absence of HEDP. The pretreated crystals were equilibrated for $5 \mathrm{~h}$ in a saturated gypsum solution containing $10^{-6} \mathrm{M}$ HEDP, at the same temperature, $\mathrm{pH}$ and solid/liquid ratio as prevailing during the growth experiments. When the pretreated seed crystals, after washing with a pure saturated gypsum solution, are used to initiate a growth experiment, their growth rate remains below the growth rate of the untreated crystals, even after prolonged outgrowth. This result again indicates that the HEDP inhibitor ions, once adsorbed, remain firmly attached to the crystal surface and are likely to be incorporated during the growth process. Fig. 7 further shows that the growth curves of the untreated and pretreated seed crystals during outgrowth in the presence of $10^{-6} \mathrm{M}$ HEDP practically coincide. The adsorption of HEDP onto the untreated seed crystals thus appears to be almost instantaneously completed after addition of the crystals to the inhibitor containing bulk solution at the start of the growth process. Similar results have been described in more detail for the adsorption of polycarboxylate inhibitors [10].

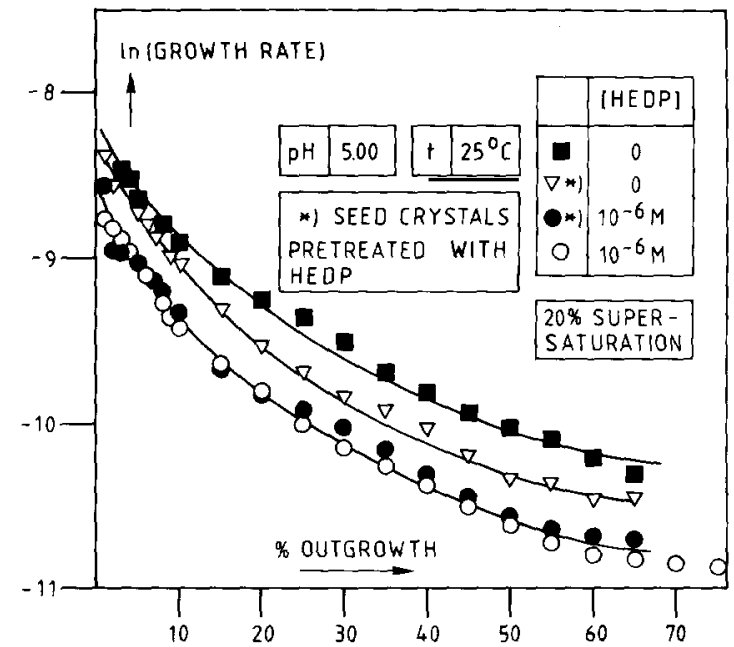

Fig. 7. Comparison of growth rates measured for "normal" seed crystals and seed crystals pretreated with HEDP, during outgrowth in the presence and in the absence of HEDP; natural logarithm of the normalized overall growth rate versus the outgrowth of the seed crystals.

\section{Conclusions}

Differences in inhibitor effectiveness between two phosphonates with a different molecular structure, as well as differences in inhibitor effectiveness of a given concentration of one phosphonate at difference $\mathrm{pH}$ values, can be correlated with a different surface coverage of the gypsum crystals with adsorbed phosphonate ions. With increasing ionic strength and decreasing $\mathrm{pH}$ values, however, deviations from this rule occur due to a gradual change in adsorption mechanism. In case of low-affinity adsorption, which not involves strong coordinative bonding with the calcium ions in the gypsum surface, the surface coverage cannot be used as a measure for the phosphonate inhibitor effectiveness.

Relatively low molecular weight inhibitors like AMDP, HEDP and TENTMP, are preferentially adsorbed at the active growth sites, provided by the microsteps upon the gypsum crystal surface. Blockage of the growth process occurs, when the total microstep length is covered with adsorbed phosphonate ions.

When adsorption experiments are performed on dry gypsum crystals, the surface coverages measured at very low inhibitor concentrations can be slightly enhanced due to Ostwald-ripening effects.

The phosphonate adsorption process is irreversible and seems to be largely completed within a few minutes.

For growth experiments performed at low phosphonate inhibitor concentrations of about $10^{-6} \mathrm{M}$, depletion of the bulk solution by AMDP or HEDP adsorption onto the seed crystal surface is most unlikely because of the low solid/liquid ratio and the low surface coverage involved. The inhibitor concentration of the bulk solution can, however, be slightly reduced by incorporation of adsorbed phosphonate ions into the growing crystals.

\section{Acknowledgements}

The authors are indebted to Professor P. Bennema for his continuous interest in their work and 
the many stimulating discussions. They are most grateful to W.G.J. Marchee for his valuable help and advice. They also wish to thank R. den Breejen, H. van der Giesen, R. Hokke and Y.F. Rody for their experimental assistance.

\section{References}

[1] G.M. van Rosmalen, Chem. Eng. Commun. 20 (1983) 209.

[2] A.D. Randolph and D.E. Vaden, AIChE Symp. Ser. 80 (1984) 110.

[3] W.H. Leung and G.H. Nancollas, J. Crystal Growth 44 (1978) 163.

[4] J.S. Gill and G.H. Nancollas, Corrosion 37 (1981) 120.

[5] M.P.C. Weijnen, W.G.J. Marchée and G.M. van Rosmalen, Desalination 47 (1983) 81.

[6] M.P.C. Weijnen, M.C. van der Leeden and G.M. van Rosmalen, in: Proc. Geochemistry of Earth Surface and Processes of Mineral Formation, Granada, 1986, Ed. R. Rodriquez.

[7] G.M. van Rosmalen and Y.F. Rody, unpublished results, 1984.

[8] A.E. Austin, J.P. Miller, D.A. Vaughan and J.F. Kircher, Desalination 16 (1975) 345.
[9] S.W. Walinsky, B.J. Morton and J.J. O'Neill, presented at ACS Meeting, Symp. on Crystal Growth from Solution, Washington, DC, 1983.

[10] M.P.C. Weijnen and G.M. van Rosmalen, Desalination 54 (1985) 239.

[11] Hach Chemical Company, Bulletin 21133 - 1 ED (1980).

[12] O.B. Michelsen, Anal. Chem. 29 (1957) 60.

[13] G.A. Hulett, Z. Physik. Chem. 47 (1904) 357.

[14] G.M. van Rosmalen, P.J. Daudey and W.G.J. Marchée, J. Crystal Growth 52 (1981) 801.

[15] M.P.C. Weijnen and G.M, van Rosmalen, in: Industrial Crystallization 84, Eds. S.J. Jančćc and E.J. de Jong (Elsevier, Amsterdam, 1984).

[16] B.L. Barnett and L.C. Strickland, Acta Cryst. B35 (1979) 1212.

[17] G.A. Hulett, Z. Physik. Chem. 37 (1901) 385.

[18] M.L. Dundon and E. Mack, J. Am. Chem. Soc. 45 (1923) 2479.

[19] M.R. Christoffersen, J. Christoffersen, M.P.C. Weijnen and G.M. van Rosmalen, J. Crystal Growth 58 (1982) 585.

[20] M.P.C. Weijnen, G.M. van Rosmalen, P. Bennema and K. Rijpkema, J. Crystal Growth, submitted.

[21] M.C. Cafe and I.D. Robb, J. Colloid Interface Sci. 86 (1982) 411

[22] P.A. Williams, R. Harrop, G.O. Phillips, G. Pass and I.D Robb, J. Chem. Soc., Faraday Trans. I, 78 (1982) 1733.

[23] W.L. Marshall and R. Slusher, J. Phys. Chem. 70 (1966) 4015. 